\title{
Phenotypic relationships, genetic parameters, genome-wide associations, and identification of potential candidate genes for ketosis and fat-to-protein ratio in German Holstein cows
}

\author{
S.-L. Klein, ${ }^{1 *}$ C. Scheper, ${ }^{1}$ K. Brügemann, ${ }^{1}$ H. H. Swalve, ${ }^{2}$ and S. König ${ }^{1}$ \\ ${ }^{1}$ Institute of Animal Breeding and Genetics, Justus-Liebig-University Gießen, 35390 Gießen, Germany \\ ${ }^{2}$ Institute of Agricultural and Nutritional Sciences, Martin Luther University Halle-Wittenberg, 06120 Halle (Saale), Germany
}

\section{ABSTRACT}

Energy demand for milk production in early lactation exceeds energy intake, especially in high-yielding Holstein cows. Energy deficiency causes increasing susceptibility to metabolic disorders. In addition to several blood parameters, the fat-to-protein ratio (FPR) is suggested as an indicator for ketosis, because a FPR $>1.5$ refers to high lipolysis. The aim of this study was to analyze phenotypic, quantitative genetic, and genomic associations between FPR and ketosis. In this regard, 8,912 first-lactation Holstein cows were phenotyped for ketosis according to a veterinarian diagnosis key. Ketosis was diagnosed if the cow showed an abnormal carbohydrate metabolism with increased content of ketone bodies in the blood or urine. At least one entry for ketosis in the first $6 \mathrm{wk}$ after calving implied a score $=1$ (diseased); otherwise, a score $=0$ (healthy) was assigned. The FPR from the first test-day was defined as a Gaussian distributed trait (FPRgauss), and also as a binary response trait (FPRbin), considering a threshold of FPR $=1.5$. After imputation and quality controls, 45,613 SNP markers from the 8,912 genotyped cows were used for genomic studies. Phenotypically, an increasing ketosis incidence was associated with significantly higher FPR, and vice versa. Hence, from a practical trait recording perspective, first test-day FPR is suggested as an indicator for ketosis. The ketosis heritability was slightly larger when modeling the pedigree-based relationship matrix (pedigree-based: 0.17; SNP-based: 0.11). For FPRbin, heritabilities were larger when modeling the genomic relationship matrix (pedigree-based: 0.09; SNP-based: 0.15). For FPRgauss, heritabilities were almost identical for both pedigree and genomic relationship matrices (pedigree-based: 0.14; SNP-based: 0.15). Genetic correlations between ketosis with FPRbin and FPRgauss

Received January 1, 2019.

Accepted March 14, 2019

*Corresponding author: sarah.klein@agrar.uni-giessen.de using either pedigree- or genomic-based relationship matrices were in a moderate range from 0.39 to 0.71 . Applying genome-wide association studies, we identified the specific SNP rs109896020 (BTA 5, position: $115,456,438 \mathrm{bp})$ significantly contributing to ketosis. The identified potential candidate gene $P A R V B$ in close chromosomal distance is associated with nonalcoholic fatty liver disease in humans. The most important SNP contributing to FPRbin was located within the DGAT1 gene. Different SNP significantly contributed to ketosis and FPRbin, indicating different mechanisms for both traits genomically.

Key words: ketosis, fat-to-protein ratio, genetic parameter, genome-wide association, potential candidate gene

\section{INTRODUCTION}

During early lactation, nutrient demand for milk production exceeds the slow increase in DMI, causing a negative energy balance (NEB). Energy deficiency is a major component explaining susceptibility to metabolic disorders, such as ketosis (KET; Buttchereit et al., 2012). Ketosis induces further cost-intensive diseases (e.g., metritis, mastitis, laminitis, or displaced abomasum; Suthar et al., 2013). During the NEB period, ketone bodies serve as short-term energy sources for several organs (Robinson and Williamson, 1980). However, excessive concentrations of circulating ketone bodies cause ketoacidosis and an increase of ketone bodies in blood, milk, and urine (Bashir et al., 2016). To overcome energy deficiency periods, fat mobilization from body fat depots is associated with increasing milk fat synthesis. Furthermore, reduced feed intake in the first third of lactation causes insufficient ruminal bacteria protein production, implying a decline in milk protein content (Gürtler and Schweigert, 2005). Hence, a fat-to-protein ratio (FPR) larger than 1.5 refers to high lipolysis. The trait FPR is available on the basis of routinely recorded test-day data, and consequently, suggested as a KET indicator (Heuer et al., 1999). 
Phenotypically, Kessel et al. (2008) analyzed metabolic processes in high-yielding Red Holstein cows during the transition period. The cows under study were kept under identical housing and feeding systems. They clustered the cows according to their ketone body concentration in plasma, and identified significant cow differences for metabolite concentrations (e.g., BHB, acetone, glucose) and for hormone levels. Such individual differences for cows kept in the same herd environment suggest genetic or epigenetic influence on physiological mechanisms coping with metabolic stress (Kessel et al., 2008).

Quantitative-genetic heritabilities for KET ranged between $0.01(\mathrm{SE}=0.006)$ and $0.14(\mathrm{SE}=0.03)$ for Holstein cows, depending on the genetic-statistical modeling approach (Pryce et al., 2016). In most studies, clinical KET cases were the trait basis for genetic parameter estimations because clinical cases are quite easy to detect (Pryce et al., 2016). Nevertheless, consideration of more precise phenotypes in genetic analyses (i.e., of subclinical KET cases) contribute to increasing incidence rates and more accurate breeding value estimations. Detection of subclinical KET implies detailed recording of biomarkers (e.g., ketone body concentrations in blood or in milk; König and May, 2018). As an alternative, test-day FPR is suggested for indirect selection strategies on subclinical KET (van Knegsel et al., 2010). Koeck et al. (2013) estimated a heritability of 0.15 ( $\mathrm{SE}=0.015$ ) for Gaussian FPR, 0.07 ( $\mathrm{SE}=$ 0.012 ) for binary FPR, and a favorable genetic correlation between FPR and KET of 0.35 ( $\mathrm{SE}=0.16$ ).

So far, only a limited number of studies have focused on genome-wide association studies (GWAS) and potential candidate gene identification for KET. Parker Gaddis et al. (2018) estimated SNP effects for producer-recorded KET, including 1,750 medium-density genotyped US Jersey cows. They identified SNP significantly associated with KET on BTA 10, 11, 14, and 23. Genes involved in insulin regulation, lipid metabolism, and immune response were located in close distance to the associated SNP markers (Parker Gaddis et al., 2018). The large number of identified significant SNP indicate that KET is a polygenetic trait, influenced by numerous genomic regions. These results are confirmed by Kroezen et al. (2018), who identified 6 KET candidate genes on BTA 2, 3, 13, 27, and 29 involved in fatty acid metabolism, gluconeogenesis, and citric acid cycle. Considering 248 genotyped Holstein cows, Tetens et al. (2015) conducted a GWAS for the KET indicator, ratio of glycerophosphocholin to phosphocholine, in milk. Via ongoing candidate gene and sequence analyses, Tetens et al. (2015) identified a QTL on BTA 25 for the ratio of glycerophosphocholine to phosphocholine.
The aim of the present study was to study phenotypic, quantitative genetic, and genomic associations between first test-day FPR and KET in first-lactation German Holstein cows, based on a large data set of genotyped cows from large scale co-operator herds. This aim includes (1) the application of generalized linear mixed models to infer phenotypic trait associations, (2) the estimation of pedigree- and SNP-based (co) variance components, (3) GWAS to detect significantly associated SNP markers, and (4) the annotation and physiological explanation of potential candidate genes.

\section{MATERIALS AND METHODS}

\section{Data}

Phenotypes. We considered 8,912 first-lactation German Holstein cows kept in 27 large-scale co-operator herds, located in the federal states of MecklenburgWest Pommerania and Brandenburg, Germany. Calving dates of cows spanned the years 2014 to 2016. Health data recording including KET was accomplished by veterinarians within the first $6 \mathrm{wk}$ after calving, using an electronic recording systems. The recording system has a hierarchical structure with several disease levels (from overall disease categories up to specific diseases), following the International Committee for Animal Recording diagnosis key (Stock et al., 2013). According to the recording guidelines, a KET diagnosis reflects disturbance of carbohydrate metabolism with increased content of ketone bodies in blood or urine (measured via handheld ketometers or urine test strips). Only herds with at least one KET entry in the 6 -wk interval were considered. Cows with at least one KET diagnosis during the 6-wk interval were coded as diseased (KET $=1$ ), whereas cows without any KET diagnosis in the 6 -wk interval were coded as healthy $(\mathrm{KET}=0)$. Cows with a culling date within the 6 -wk interval have "no further chance" to get the disease. Hence, these cows with no KET entry until the culling date are considered as healthy. This is a general problem with health data (i.e., cullings within the defined time period). The early lactation interval definition was used for other disease categories, such as claw disorders (König et al., 2005). Nevertheless, KET has only a minor effect on statistic or genetic parameter estimations because (1) KET is diagnosed very early after calving $(60 \%$ within the first $10 \mathrm{~d}$ after calving), and (2) the number of cullings is very small within the 6 -wk interval after calving. Most of the cullings occur later in lactation. In our data set, only $0.75 \%$ of the cows diagnosed as healthy had a culling date before the second test-day. Descriptive statistics for KET are given in Table 1. Most of the KET- 
Table 1. Descriptive statistics for ketosis (KET) and fat-to-protein ratio (FPR) of first lactating Holstein cows

\begin{tabular}{lc}
\hline Characteristic & $\begin{array}{c}\text { Analyzed } \\
\text { data }\end{array}$ \\
\hline Total no. of herds & 27 \\
Total no. of animals & 8,912 \\
Mean no. of cows per herd & 330 \\
Healthy animals KET, no. $(\%)$ & $8,750(98.18)$ \\
Diseased animals KET, no. $(\%)$ & $162(1.82)$ \\
Healthy animals FPR $\leq 1.5$, no. $(\%)$ & $7,688(86.27)$ \\
Diseased animals FPR $>1.5$, no. $(\%)$ & $1,224(13.73)$ \\
\hline
\end{tabular}

diagnosed cows ( $\sim 80 \%$ of the cows) were diagnosed for another disease, such as claw disorders or mastitis.

Test-day records included milk yield, fat percentage, protein percentage, FPR, and SCS from the first test-day after calving. The first test-day was within the period from 5 to 42 DIM. Descriptive statistics for all test-day traits are given in Table 2. According to Hein et al. (2018), extremely low values for milk yield (2 $\mathrm{kg}$ ) were considered because very strong KET cases might cause a sudden milk yield decline. The data editing threshold for milk yield as used in official genetic evaluations is $5 \mathrm{~kg}$, but only 14 test-day records were in the range from 2 to $5 \mathrm{~kg}$. Somatic cell count was logtransformed into SCS as follows: SCS $=\log _{2}(\mathrm{SCC} / 100)$ +5 (Ali and Shook, 1980). The number of SCS records was slightly smaller than for milk yield or percentage traits because of a few missing records for SCC.

Furthermore, we calculated lactation persistency, using the formula by Visscher and Mason (2016):

$$
\left.\begin{array}{l}
\text { Persistency }(\%)= \\
{\left[-\frac{\left[\begin{array}{l}
\text { milk yield }(\mathrm{kg}) \text { earlier test-day } \\
- \text { milk yield }(\mathrm{kg}) \text { later test-day }
\end{array}\right] \times \frac{30 \mathrm{~d}}{\text { days between }}}{\text { test-days }}\right.} \\
\text { milk yield }(\mathrm{kg}) \text { earlier test-day }
\end{array}\right]
$$

$\times 100$.

In this regard, the earlier test-day was test-day 3 , and the later test-day was test-day 5 . Test-day 3 was within 50 and 140 DIM, and test-day 5 reflected the lactation period from 150 to 220 DIM. Data editing excluded records with persistency values lower than $65 \%$ or larger than $120 \%$.

Fat-to-protein ratio was defined as a Gaussian distributed trait (FPRgauss) and as a binary response trait (FPRbin). A FPR $>1.5$ at the first test-day implied a score of 1 for FPRbin (diseased); otherwise, a score of 0 (healthy) was assigned. The incidences for the binary trait definitions are in Table 1 .

Genotypes. A total of 2,374 cows were genotyped with the Illumina BovineSNP50 v2 BeadChip (Illumina Inc., San Diego, CA), and 6,538 cows with the EuroGenomics $10 \mathrm{~K}$ chip. Low-density genotypes were imputed by the project partner VIT (Vereinigte Informationssysteme Tierhaltung w.V, Verden, Germany) to the $50 \mathrm{~K}$ panel, applying the algorithm as outlined by Segelke et al. (2012). The SNP data set considered 45,613 SNP from the 8,912 phenotyped cows. Quality control of the genotype data was performed using the software package PLINK (Purcell et al., 2007). The SNP with more than $1 \%$ missing genotype data, with a minor allele frequency lower than 5\% and deviation from HardyWeinberg equilibrium $\left(P<1 \times 10^{-8}\right)$, were discarded. Thus, due to different cows in case and control groups for both traits, 40,993 and 41,017 SNP from the 8,912 cows were available for genomic studies for KET and FPRbin, respectively.

\section{Statistical Models}

Phenotypic Associations Between Fat/Protein Ratio and Ketosis. A generalized linear mixed model (GLMM) with a binomial distribution and a logit link function was applied to analyze the effect of FPRbin on binary KET. For this purpose, the Glimmix procedure in SAS (version 9.4, SAS Institute Inc., Cary, NC) was used. The statistical model 1 was

$$
\begin{aligned}
& \operatorname{logit}(\pi)=\log \left[\pi_{\text {rstuv }} /\left(1-\pi_{\text {rstuv }}\right)\right]=\varphi+\operatorname{Herd}_{\mathrm{r}} \\
& + \text { CYear }_{\mathrm{s}}+\text { CMon }_{\mathrm{t}}+\mathrm{CAge}_{\mathrm{u}}+\text { FPRbin }_{\mathrm{v}}, \quad[\text { model } 1]
\end{aligned}
$$

where $\pi_{\text {rstuv }}=$ probability of a KET occurrence; $\varphi=$ overall mean effect; Herd $_{\mathrm{r}}=$ fixed herd effect (27 herds); CYear $_{\mathrm{s}}=$ fixed effect of calving year $(3 \mathrm{yr}, 2014-2016)$; $\mathrm{CMon}_{\mathrm{t}}=$ fixed effect of calving month (12 mo); CA$\mathrm{ge}_{\mathrm{u}}=$ covariate age at first calving (linear regression); FPRbin $_{\mathrm{v}}=$ fixed effect of FPRbin $(2$ classes, FPR $\leq 1.5$ or FPR >1.5).

The recursive effect (i.e., of KET on the Gaussian distributed test-day traits milk yield, fat percentage, protein percentage, FPR, SCS, and persistency) also was analyzed using the Glimmix procedure in SAS (SAS Institute Inc.). In this regard, a GLMM with an identity link function was defined. The statistical model 2 was

$$
\begin{aligned}
& \mathrm{y}_{\mathrm{ijklm}}=\varphi+\operatorname{Herd}_{\mathrm{i}}+\mathrm{CYear}_{\mathrm{j}}+\mathrm{CMon}_{\mathrm{k}}+\mathrm{CAge}_{\mathrm{l}} \\
& +\mathrm{KET} \times \text { Diffdat }_{\mathrm{m}}+\mathrm{e}_{\mathrm{ijklm}}, \quad[\text { model } 2]
\end{aligned}
$$


Table 2. Descriptive statistics for first official test-day traits of first lactating Holstein cows

\begin{tabular}{|c|c|c|c|c|c|}
\hline Trait & $\begin{array}{l}\text { No. of } \\
\text { records }\end{array}$ & Mean & SD & Minimum & Maximum \\
\hline Milk yield (kg) & 8,912 & 28.88 & 6.58 & 2 & 53.8 \\
\hline Fat percentage $(\%)$ & 8,912 & 4.10 & 0.78 & 1.62 & 9.26 \\
\hline Protein percentage $(\%)$ & 8,912 & 3.22 & 0.32 & 2.24 & 4.89 \\
\hline Fat-to-protein ratio & 8,912 & 1.28 & 0.23 & 0.43 & 2.91 \\
\hline Fat-to-protein ratio $>1.5$ & 1,224 & 1.70 & 0.21 & 1.50 & 2.91 \\
\hline SCS & 8,893 & 4.85 & 1.69 & 0.94 & 11.64 \\
\hline Persistency (\%) & 8,549 & 99.14 & 5.09 & 66.87 & 119.86 \\
\hline
\end{tabular}

where $\mathrm{y}_{\mathrm{ijklm}}=$ observation for the test-day traits; Herd $_{i}=$ fixed herd effect $(27$ herds $) ;$ CYear $_{j}=$ fixed effect of calving year $(3 \mathrm{yr}, 2014-2016) ; \mathrm{CMon}_{\mathrm{k}}=$ fixed effect of calving month $(12 \mathrm{mo}) ; \mathrm{CAge}_{1}=$ covariate age at first calving (linear regression); KET $\times$ Diffdat $_{\mathrm{m}}=$ combined fixed effect of KET (healthy or diseased) $x$ period between the first test-date and the KET diagnosis date $[9$ classes considering the following periods: 31 to $21 \mathrm{~d}$ ( $\mathrm{n}=5$ cows), 20 to $11 \mathrm{~d}(\mathrm{n}=8$ cows), 10 to $5 \mathrm{~d}(\mathrm{n}=13 \mathrm{cows}), 4$ to $1 \mathrm{~d}(\mathrm{n}=15$ cows $)$ before the diagnosis date; 0 to $4 \mathrm{~d}$ ( $\mathrm{n}=13$ cows), 5 to $10 \mathrm{~d}$ ( $\mathrm{n}=$ 33 cows), 11 to $20 \mathrm{~d}$ ( $\mathrm{n}=43$ cows), and 21 to $31 \mathrm{~d}$ ( $\mathrm{n}=$ 32 cows) after the diagnosis date; and a "dummy" class for healthy cows $(\mathrm{n}=8,750$ cows $)]$; and $\mathrm{e}_{\mathrm{ijklm}}=$ random residual. A multiple comparison adjustment for the effect of the KET $\times$ Diffdat effect was accomplished using the Dunnett correction as implemented in the Glimmix procedure (Dunnett, 1955).

Pedigree- and Genomic-Based Genetic Parameter Estimation. Pedigree-based variance components and heritabilities for KET and FPR were estimated using the AI-REML algorithm as implemented in the DMU software package (Madsen and Jensen, 2013). The pedigree file consisted of 93,446 animals. The GLMM (model 3) considered the same fixed effects as specified in model 1 and model 2, but we (1) excluded the explanatory variables FPRbin and KET $\times$ Diffdat, and $(2)$ we included the random additive-genetic animal effect.

Genetic correlations between KET and FPRgauss and FPRbin were estimated via bivariate linear animal models.

The SNP-based variance components, heritabilities, and genomic correlations were estimated with GCTA (Yang et al., 2011), using the GREML function. Fixed effects were identical to the pedigree-based analyses, but instead of using the pedigree-based relationship matrix, the genomic relationship matrix (GRM) was constructed.

Genome-Wide Association Studies. The data set used for FPRbin included 1,224 cases (FPR >1.5) and 7,688 controls $(\mathrm{FPR} \leq 1.5)$. Regarding KET, 162 cases (diseased) and 8,750 controls (healthy) were available. Also for GWAS, the GCTA software (Yang et al., 2011) was applied. In GCTA, we used the option mlma-loco (i.e., to perform a mixed linear model via the "leaving one chromosome out" strategy). The model 4 was

$$
\mathbf{y}_{i j}=\mathbf{X} \boldsymbol{\beta}+\mathbf{Z u}+\mathbf{S}_{k} \mathrm{~S}_{j k}+\mathbf{e}_{i j}, \quad[\text { model } 4]
$$

where $\mathbf{y}_{i j}$ represents the vector of phenotypic observations from the $i$ th cow $(i=1-8,912)$ for the $j$ th trait $(j$ $=\mathrm{KET}$ or FPRbin), $\mathbf{X}$ is the incidence matrix relating fixed effects in $\boldsymbol{\beta}$ (as specified in model 4) with observations in $\mathbf{y}_{i j}, \mathbf{Z}$ is the incidence matrix relating random additive polygenic effects in $\mathbf{u}$ with observations in $\mathbf{y}_{i j}$, $\mathbf{S}_{k}$ is the vector of genotypes for the $k$ th SNP across all animals, $\mathrm{s}_{j k}$ represents the additive effect of the $k$ th $\mathrm{SNP}$ on the $j$ th trait, and $\mathbf{e}_{i j}$ is the vector of random residual effects.

For the trait FPRbin, Manhattan plots from model 4 suggested a strong effect of the single SNP rs109421300, which is located in the DGAT1 gene. It was our intention to account for a major DGAT1 gene effect on the traits of interest. Hence, as an extension of model 4, an additional model 5 was chosen for both traits KET and FPRbin. In model 5, we considered the genotype for the DGAT1 SNP rs109421300 as a further fixed effect.

The effective number of independent $\mathrm{SNP}\left(\mathrm{N}_{\mathrm{SNP}}\right.$ $=29,548)$ was calculated using the software Genetic type 1 Error Calculator ( $\mathrm{Li}$ et al., 2012) to define the genome-wide significance level according to Bonferroni $\left(\mathbf{p B F}=0.05 / \mathrm{N}_{\mathrm{SNP}}=1.69 \mathrm{e}-06\right)$. In addition, a less conservative normative significance threshold was used to identify potential candidate SNP, considering $\mathbf{p C D}=$ 1e-04 (Kurz et al., 2019). Annotated genes located in $250 \mathrm{~kb}$ upstream or downstream from the significantly associated candidate SNP were detected using Ensembl release 91 (Zerbino et al., 2018).

\section{RESULTS AND DISCUSSION}

\section{Phenotypic Associations Between Ketosis and Test-Day Traits}

Phenotypically, increasing KET incidences were associated with significantly higher FPR $(P<0.001$, model 
[1]). The probability for KET occurrence was 3-fold higher for cows with a FPR larger than 1.5 (predicted incidence $=0.050, \mathrm{SE}=0.022)$ compared with cows with a FPR lower or equal 1.5 (predicted incidence = $0.017, \mathrm{SE}=0.007$ ). Vice versa, we also identified a significant influence of a KET diagnosis on FPR, but depending on the intervals between the test date and the diagnosis date (model [2]). Table 3 addresses the influence from KET diagnoses recorded before and after the test date. Cows with a KET diagnosis had a significant higher first test-day FPR and fat percentage compared with healthy cows. Differences between sick and healthy cows were most obvious for diagnosis days in close distance to the test-day (i.e., test-day 4 to $1 \mathrm{~d}$ before and 0 to $10 \mathrm{~d}$ after the diagnosis date). Hence, FPR is a very useful KET indicator for a dense data structure (i.e., in the optimal case for records on a daily level from automatic milking systems; Santos et al., 2018). Increased FPR and fat percentage for cows with a KET diagnosis reflect the increased milk fat synthesis caused by elevated body fat mobilization during the energy deficiency period (Bell, 1995).

Milk yield was significantly lower for KET-diagnosed cows in the period from $10 \mathrm{~d}$ before to $20 \mathrm{~d}$ after the diagnosis date (Table 3). Accordingly, Koeck et al. (2013) confirmed the decline in milk yield during the disease period for cows with a KET diagnosis. In our study, protein percentage was significantly lower from d 11 to 31 after the diagnosis date (Table 3), compared with protein percentage of healthy cows. The decrease of test-day protein percentage after the KET diagnosis date is in agreement with Duffield et al. (2009). The insufficient intake of carbohydrates in the state of NEB decreases the microbial protein synthesis, causing the decline in milk protein percentage (Gürtler and Schweigert, 2005).

Phenotypically, in our study, KET had no significant effect on SCS and persistency.

\section{Heritabilities and Genetic Correlations}

Heritabilities were 0.17 and 0.11 for KET, 0.09 and 0.15 for FPRbin, and 0.14 and 0.15 for FPRgauss, when applying pedigree-based or genomic models, respectively (Table 4, model [3]). In other studies using producer data, pedigree-based heritabilities for KET ranged between 0.02 and 0.14 (Koeck et al., 2014; Heringstad et al., 2005). In general, low heritabilities for KET might be due to the complex disease trait character, showing the phenomenon of the missing heritability (Blanco-Gómez et al., 2016). The pathogenesis of complex diseases is often regulated by intermediate phenotypes with quantitative inheritance. Blanco-Gómez et al. (2016) argued that not-detectable intermediate phenotypes explain a major part of the missing heritability. An application of more precise continuous phenotypes for KET, such as Fourier transform mid-infrared blood or milk BHB concentration, may lead to detection of subclinical cases, higher incidences, and higher heritability estimates (Belay et al., 2017). For KET, we estimated smaller SNP-based heritabilities than pedigree-based heritabilities, indicating that

Table 3. Differences for LSM of first test-day traits for the cow group comparison of ketosis healthy cows minus ketosis diseased cows [considered time periods: test-day dates before (b) or after (a) the ketosis diagnosis date]

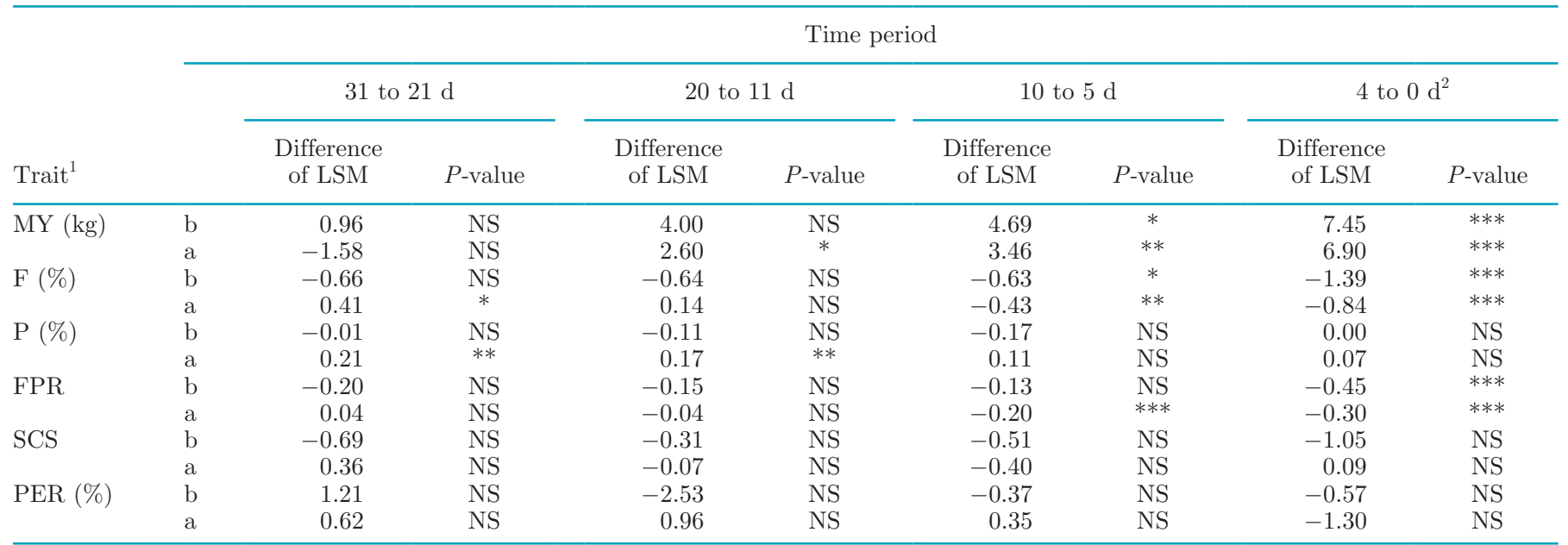

${ }^{1} \mathrm{MY}=$ milk yield; $\mathrm{F}=$ fat percentage; $\mathrm{P}=$ protein percentage; $\mathrm{FPR}=$ fat-to-protein ratio; $\mathrm{PER}=$ persistency.

${ }^{2} 4$ to $0 \mathrm{~d}$ : 4 to $1 \mathrm{~d}$ before the ketosis diagnosis date, 0 to $4 \mathrm{~d}$ after the ketosis diagnosis date.

${ }^{* * *} P \leq 0.001 ;{ }^{* *} P \leq 0.01 ;{ }^{*} P \leq 0.05$. 


\begin{tabular}{|c|c|c|c|c|}
\hline Method & Trait & KET & FPRbin & FPRgauss \\
\hline \multirow[t]{2}{*}{ Pedigree-based } & KET & $0.17(0.08)$ & $0.71(0.18)$ & $0.52(0.16)$ \\
\hline & $\begin{array}{l}\text { FPRbin } \\
\text { FPRgauss }\end{array}$ & & $0.09(0.02)$ & $0.14\left(\begin{array}{ll}0.02)\end{array}\right.$ \\
\hline \multirow[t]{3}{*}{ Genomic-based } & KET & $0.11(0.06)$ & $0.50(0.21)$ & $0.39(0.17)$ \\
\hline & FPRbin & & $0.15(0.02)$ & \\
\hline & FPRgauss & & & $0.15(0.01)$ \\
\hline
\end{tabular}

the 40,993 SNP marker panel did not fully explain the genetic trait variation. Accordingly, Yang et al. (2017) assumed smaller SNP heritabilities for complex diseases for low- or medium-density SNP chips because not all causal variants are in linkage disequilibrium with the available SNP. McNeel et al. (2017) defined KET as a wellness trait, and suggested a reduction of disease incidences and improvements of farm profitability through the implementation of direct genetic selection strategies on low heritability wellness traits.

In analogy to KET, heritabilities for FPRbin and FPRgauss were quite small (Table 4). Koeck et al. (2013) estimated a pedigree heritability of 0.15 for first test-day FPRgauss, and of 0.07 for FPRbin. We detected slight differences between the SNP- and pedigree-based heritability for FPRbin. Opposite to KET, the pedigree heritability for FPRbin (0.09) was lower than the SNP-based estimation (0.15). Low estimates for pedigree-based heritabilities point to erroneous or missing pedigree information. However, the same pedigree was used for KET and FPR genetic analyses. On the other hand, FPRgauss heritabilities were almost identical for both pedigree and genomic relationship matrices, probably due to the Gaussian trait character (Golan et al., 2014; Ge et al., 2017).

Genetic correlations between KET and FPRgauss, and between KET and FPRbin, are given in Table 4. Genetic correlations from different methods (i.e., SNP- versus pedigree-based) ranged from moderate to high levels. Genetic correlations between KET and FPRgauss were 0.39 (SNP-based) and 0.52 (pedigreebased), and 0.50 (SNP-based) and 0.71 (pedigreebased) between KET and FPRbin (model [3]). Genetic correlations based on the GRM were lower than pedigree-based genetic correlations. This refers to the missing correlation phenomenon caused by the fact that the covariance between KET and FPR was not fully explained by the used SNP marker (Momen et al., 2017). As estimates for complex traits may differ between pedigree-based and genomic approaches, Momen et al. (2017) suggested utilization of both information sources simultaneously.

\section{Genome-Wide Association Studies}

Ketosis. Applying GWAS, we identified 5 suggestively associated candidate SNP on BTA 5, 8, 9, and 15 contributing to KET (Figure 1, model [4]). Twenty-three genes in the interval of $250 \mathrm{~kb}$ surrounding the suggestive SNP were retrieved from the Ensembl database (Zerbino et al., 2018; Table 5). The SNP rs109896020 with strongest association was located on BTA 5, and 4 potential candidate genes involved in physiological processes were located in the defined interval: EFhand calcium binding domain $6(E F C A B 6)$, parvin $\beta$ $(P A R V B)$, parvin gamma $(P A R V G)$, and shisa like 1 (SHISAL1/KIAA1644). Eckel-Passow et al. (2014) identified downregulations of $E F C A B 6$ expressions in obese individuals. In their study, $E F C A B 6$ expression profiles were associated with obesity-related renal cell carcinoma in humans, suggesting a relationship with mechanisms of the lipid metabolism. The PARVB gene, located in close chromosomal distance $(61,382 \mathrm{bp})$ to the SNP rs109896020, contributes to nonalcoholic fatty liver disease in humans (Kitamoto et al., 2013). Applying a genome-wide case-control association study, a haplotype in the $P A R V B$ gene was strongly associated with nonalcoholic fatty liver disease, suggesting an involvement of $P A R V B$ in the lipid metabolism. In addition, $P A R V B$ encodes a member of the parvin family of actin-binding proteins, which inhibits the activity of integrin-linked kinase (NCBI, 2017). Integrins in turn play a crucial role regarding the progression of fibrosing liver diseases (Patsenker and Stickel, 2011). The increased influx of fatty acids in the liver, caused by the elevated body fat mobilization in the state of NEB, exceeds the metabolic capacity. An increase of the metabolic capacity contributes to hepatocyte accumulations of triglycerides and causes the fatty liver disease. Ketosis-diseased cows often have fatty livers (White, 2015), suggesting PARVB as a potential candidate gene regulating KET processes. Similarly, Wu et al. (2016) detected an association between polymorphisms in the $P A R V B$ gene with the fatty liver disease syndrome in humans. 


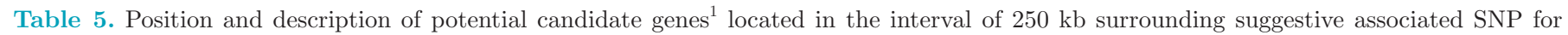
ketosis in first-lactation Holstein cows

\begin{tabular}{|c|c|c|c|c|}
\hline BTA & Start (bp) & End (bp) & Ensembl Gen ID & Description \\
\hline 5 & $115,127,019$ & $115,149,183$ & ENSBTAG00000030189 & EFCAB 6 , EF-hand calcium binding domain 6 \\
\hline 5 & $115,517,920$ & $115,572,329$ & ENSBTAG00000021978 & $P A R V B, \beta$-parvin \\
\hline 5 & $115,583,627$ & $115,608,935$ & ENSBTAG00000000805 & $P A R V G$, parvin gamma \\
\hline 5 & $115,683,472$ & $115,697,601$ & ENSBTAG00000003574 & KIAA1644 \\
\hline 8 & $9,276,105$ & $9,481,183$ & ENSBTAG00000017232 & KIF13B, kinesin family member $13 \mathrm{~B}$ \\
\hline 8 & $9,500,635$ & $9,691,300$ & ENSBTAG00000010819 & $H M B O X 1$, homeobox containing 1 \\
\hline 8 & $9,692,513$ & $9,781,880$ & ENSBTAG00000008845 & INTS9, integrator complex subunit 9 \\
\hline 9 & $64,879,264$ & $64,929,935$ & ENSBTAG00000011330 & ENSBTAG00000011330 \\
\hline 9 & $65,241,868$ & $65,241,949$ & ENSBTAG00000047184 & ENSBTAG00000047184 \\
\hline 9 & $65,003,566$ & $65,003,656$ & ENSBTAG00000044914 & U6, U6 spliceosomal RNA \\
\hline 15 & $44,335,342$ & $44,460,360$ & ENSBTAG00000005356 & ST5, suppression of tumorigenicity 5 \\
\hline 15 & $44,175,366$ & $44,203,114$ & ENSBTAG00000013366 & NRIP3, nuclear receptor interacting protein 3 \\
\hline 15 & $44,199,004$ & $44,199,141$ & ENSBTAG00000038873 & U3, small nucleolar RNA U3 \\
\hline 15 & $44,215,628$ & $44,234,314$ & ENSBTAG00000008310 & TMEM9B, TMEM9 domain family member B \\
\hline 15 & $44,237,476$ & $44,237,790$ & ENSBTAG00000004771 & ENSBTAG000000047r1 \\
\hline 15 & $44,246,871$ & $44,247,423$ & ENSBTAG00000032339 & $A S C L 3$, achaete-scute family bHLH transcription factor 3 \\
\hline 15 & $44,250,291$ & $44,260,216$ & ENSBTAG00000001917 & C11orf16, chromosome 11 open reading frame 16 \\
\hline 15 & $44,260,212$ & $44,267,703$ & ENSBTAG00000001922 & $A K I P 1$, A-kinase interacting protein 1 \\
\hline 15 & $44,469,327$ & $44,472,127$ & ENSBTAG00000005349 & $R P L 27 A, 60 \mathrm{~S}$ ribosomal protein L27a \\
\hline 15 & $44,469,600$ & $44,469,728$ & ENSBTAG00000042335 & SNORA3, small nucleolar RNA SNORA3/SNORA45 family \\
\hline 15 & $44,470,841$ & $44,470,970$ & ENSBTAG00000042354 & SNORA3, small nucleolar RNA SNORA3/SNORA45 family \\
\hline 15 & $44,500,912$ & $44,530,712$ & ENSBTAG00000020890 & TRIM66, tripartite motif containing 66 \\
\hline 15 & $44,552,624$ & $44,726,438$ & ENSBTAG00000011910 & STK33, serine/threonine kinase 33 \\
\hline
\end{tabular}

${ }^{1}$ Ensembl release 91 (Zerbino et al., 2018).

Interestingly, the only suggestively associated marker on BTA 5 for KET is the SNP rs109896020. The Manhattan-plot for KET (Figure 1) indicates that no other polymorphism close to SNP rs109896020 surpasses the candidate threshold. Probably, variation generated by the causal variants is not fully explained by genotyped SNP from the medium-density chip. Cow genotyping using a denser SNP panel, or utilization of sequence data, might contribute to the identification of a larger number of significantly associated genetic markers within this specific chromosomal segment.
On BTA 8, the SNP rs41860668 and rs41859808 surpassed the candidate threshold. One of the 3 genes including these $2 \mathrm{SNP}$ is the homeobox containing 1 (HMBOX1) gene. In humans, Chen et al. (2006) identified associations between HMBOX1 polymorphisms and type 2 diabetes. Furthermore, Dai et al. (2011) showed that $H M B O X 1$ is highly expressed in the hepatic tissue, which plays an important role in the glycometabolism (Rui, 2014). Moreover, a mutation in the HNF1 homeobox A (HNF1A) gene was associated with insulin-dependent diabetes mellitus and maturity-onset

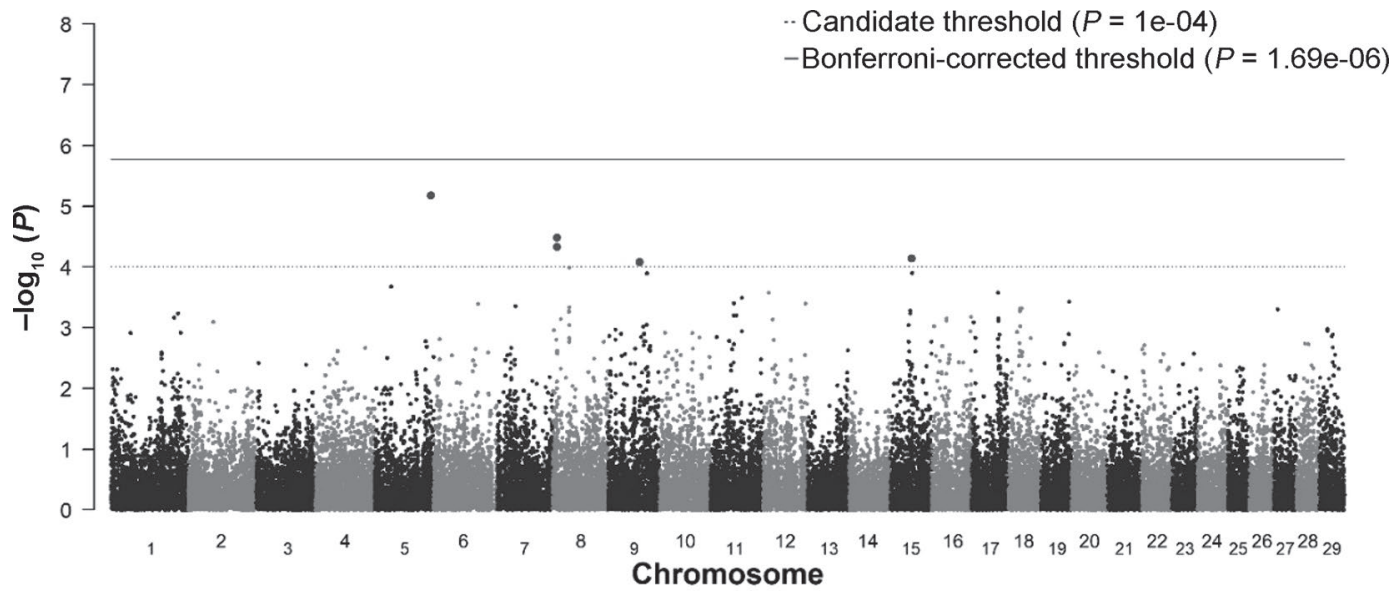

Figure 1. Manhattan plot for SNP effects for ketosis of first-lactation Holstein cows. 
diabetes (Owen and Hattersley, 2001). Ketosis is closely related to diabetes since diabetes is the most common pathological cause of elevated blood ketones (Preeti and Sushil, 2016). Due to the effect of HMBOX1 on diabetes, and due to the associations between diabetes with NEB and KET, we hypothesize that HMBOX1 also plays a crucial role in the progression of KET. On BTA 9, SNP rs42858549 was suggestively associated with KET. One of the 3 genes including this specific SNP is protein coding ENSBTAG00000011330. A disease-associated ortholog of ENSBTAG00000011330 was significantly increased in diabetic rats, and in consequence, associated with diabetes mellitus (Schmatz et al., 2009).

On BTA 15, 13 genes were located in the defined interval in close distance to the candidate SNP rs109536046. The genes tripartite motif containing 66 (TRIM66), serine/threonine kinase 33 (STK33), 60S ribosomal protein L27a $(R P L 27 A)$, and suppression of tumorigenicity 5 (ST5) influence diabetes mellitus and body mass in humans. Lau et al. (2017) focused on next generation sequencing and identified genetic susceptibility to type 2 diabetes. In humans, functional annotations were close to the potential candidate genes TRIM66 and STK33. Furthermore, Rask-Andersen et al. (2013) reported effects of the STK33 polymorphism rs4929949, located within a $47 \mathrm{~kb}$ haplotype block, on the body mass index in children. The STK33 is located in in close distance to TRIM66, RPL27A, and ST5, which are all located within a $200 \mathrm{~kb}$ segment surrounding the marker rs4929949. Obesity or a large body mass index are associated with diabetes, and with high frequency, obesity and diabetes occur together (Golay and Ybarra, 2005). Recently, Kroezen et al.
(2018) identified 6 genes involved in lipid, ketogenic, or glucose metabolism, or in the citric acid cycle. The SNP and genes identified in our study were different from those detected by Kroezen et al. (2018).

Fat/Protein Ratio. In total, 24 genome-wide significant SNP ( $\mathrm{pBF}=1.69 \mathrm{e}-06)$ and 30 suggestive SNP $(\mathrm{pCD}=1 \mathrm{e}-04)$ were detected for FPRbin on different chromosomes [i.e., 1 SNP on BTA 3, BTA 4, BTA 24, BTA 28, and BTA 29; 2 SNP on BTA 13; 3 SNP on BTA 9; 4 SNP on BTA 27; and 40 SNP on BTA 14; Figure 2, model 4]. The Manhattan-plot (Figure 2) illustrates that the majority of the SNP was located on BTA 14. Due to the large number of detected SNP for FPRbin, we only focused on gene annotations for significantly associated SNP. As expected, the most significant associated SNP rs109421300 $\left[-\log _{10}(P)=\right.$ 17.19] was located within the diacylglycerol O-acyltransferase 1 (DGAT1) gene. The DGAT1 and genes in the DGAT1 cluster [e.g., scratch family transcriptional repressor 1 (SCRT1), heat shock transcription factor 1 (HSF1), protein phosphatase 1 regulatory subunit 16A (PPP1R16A), forkhead box H1 (FOXH1), cysteine and histidine rich 1 ( $C Y H R 1$ ), plectin (PLEC), lymphocyte antigen 6 family member $\mathrm{H}(L Y 6 H)$, rhophilin Rho GTPase binding protein 1 (RHPN1), chromosome 14 open reading frame, human C8orf33 (C14H8orf33), kinesin family member $\mathrm{C} 2$ (KIFC2), and tonsoku like, DNA repair protein $(T O N S L)]$, are associated with milk yield, fat or protein percentage, and fatty acid composition in Holstein cows ( $\mathrm{Li}$ et al., 2014; Bovenhuis et al., 2016). Results from random regression analyses (Oikonomou et al., 2009) implied significant effects of a polymorphism in the DGAT1 gene on energy and metabolic traits in Holstein cows. The influenced traits

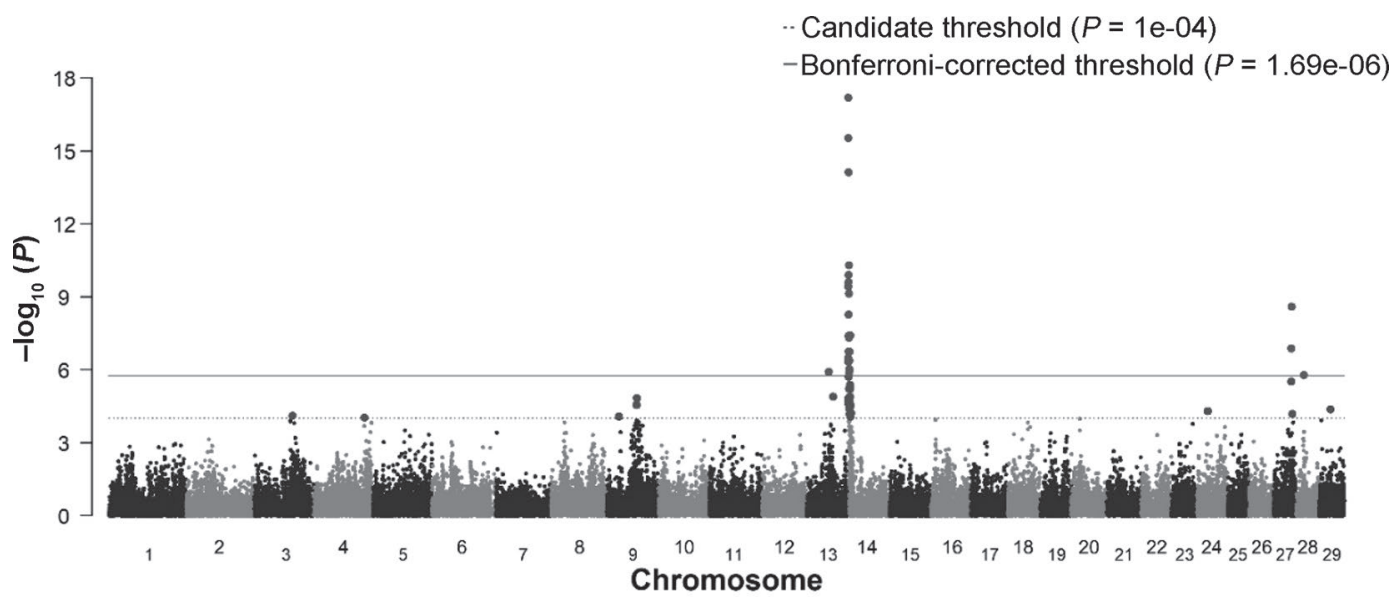

Figure 2. Manhattan plot for SNP effects for binary distributed first test-day fat-to-protein ratio of first-lactation Holstein cows. 


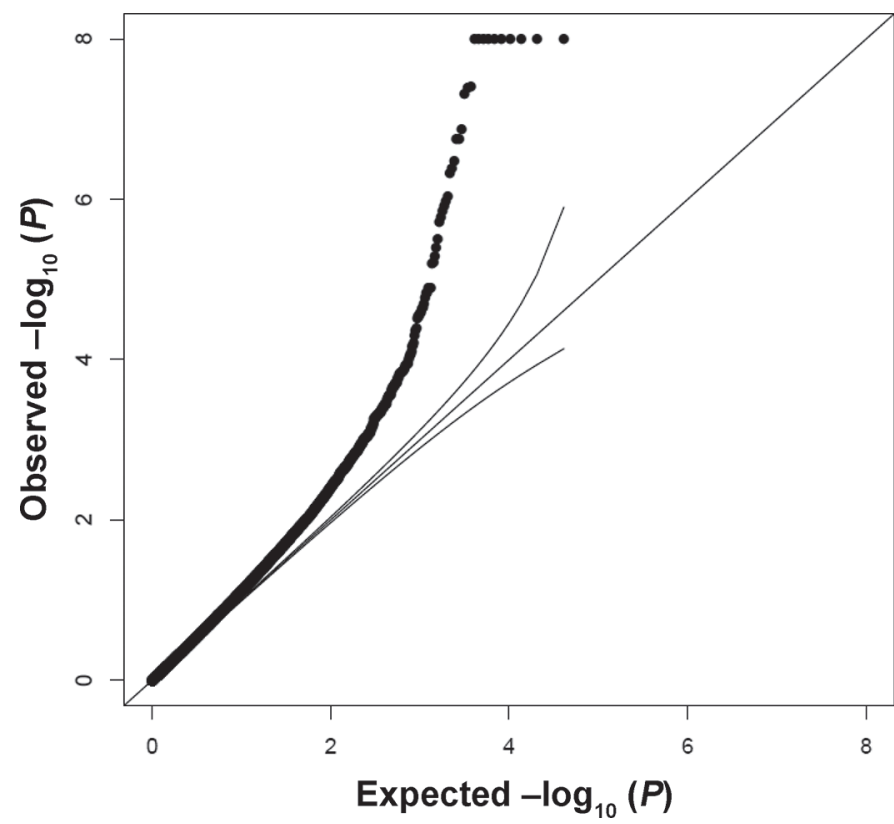

Figure 3. Quantile-quantile plot for binary distributed first testday fat-to-protein ratio.

in first-lactation cows were BCS, energy balance, and serum levels of fatty acids during the first 4 wk after calving.

The 2 SNP $\operatorname{rs} 110519353\left[-\log _{10}(P)=8.60\right]$ and rs109599512 $\left[-\log _{10}(P)=6.88\right]$ on BTA 27 surpassed the Bonferroni corrected threshold and are located in a segment for a QTL influencing milk fat percentage on BTA 27 (Littlejohn et al., 2014). Variants of the gene glycerol-3-phosphate acyltransferase 4 (AGPAT6; AGPAT6 includes the SNP rs110519353) are associated with milk fat percentage in Holstein-Friesian $\times$ Jersey crossbreed, indicating involvement in lipid metabolism
(Littlejohn et al., 2014). In addition, the SNP rs43088681 on BTA 13 (position: 46,239,050 bp) was significantly associated with FPRbin. A polymorphism in the adenosine deaminase RNA-specific B2 (ADARB2) gene, located in the specified interval surrounding this SNP, is significantly associated with diabetes-related traits in humans (Oguro et al., 2012). Individuals with a particular genotype for ADARB2 polymorphism showed high serum triglyceride and serum adiponectin levels, implying a contribution of $A D A R B 2$ in energy metabolism.

The quantile-quantile (QQ) plot for FPRbin (Figure 3) illustrates a strong deviation from the diagonal, possibly due to the strong effect of the most significant associated SNP rs109421300 located in the DGAT1 gene. In consequence, to verify this hypothesis, we included the SNP genotype for rs109421300 as an additional fixed effect in our association analyses for FPRbin (see model [5]). The Manhattan and QQ plots for FPRbin after DGAT1 correction display the expected results. Previously detected significant associated SNP on BTA 14 were eliminated due to the DGAT1 correction (Figure 4). Accordingly, the QQ plot indicates less deviation from the diagonal (Figure 5). In a next step, we additionally considered the SNP genotype for rs109421300 as a fixed effect in the association analysis for KET (model [5]). The Manhattan- and QQ plots for KET with or without DGAT1 correction display no differences, suggesting that this SNP only has a substantial effect on FPR, but not on KET. This finding indicates that rs109421300 is not involved in milk fat synthesis processes in the states of NEB and KET (i.e., transformations from mobilized body fat depots into milk fat), but contributes to the DGAT1 effect on high fat percentages.

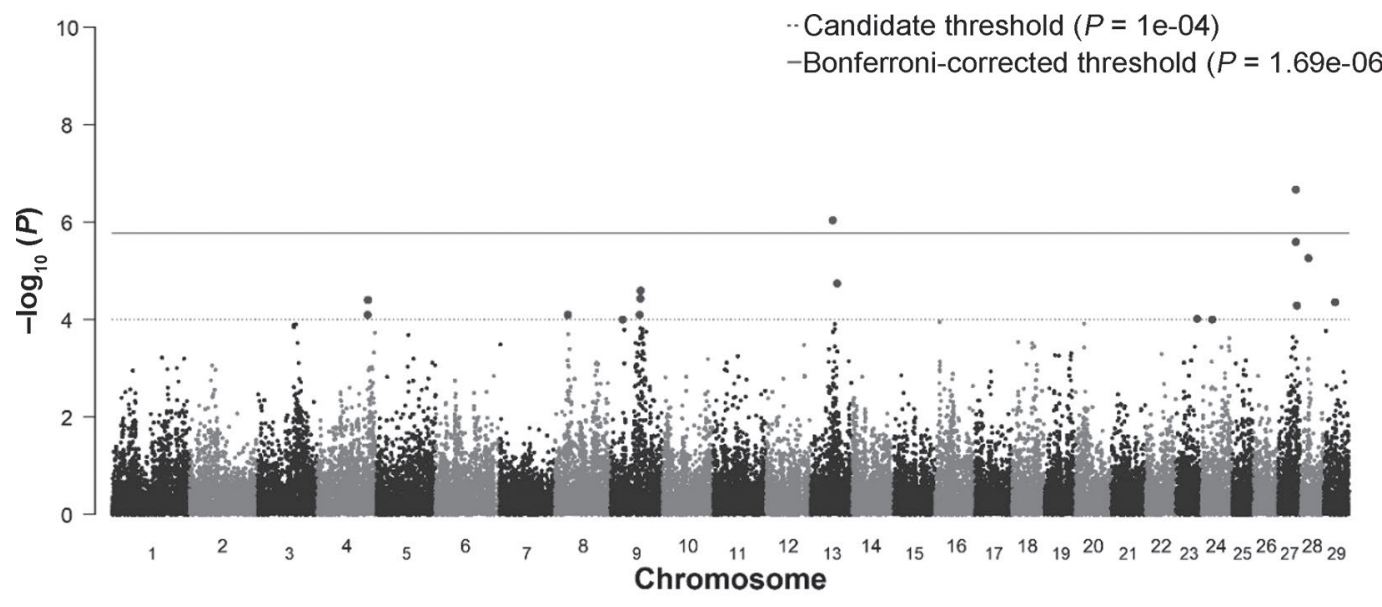

Figure 4. Manhattan plot for SNP effects for binary distributed first test-day fat-to-protein ratio after DGAT1 correction. 


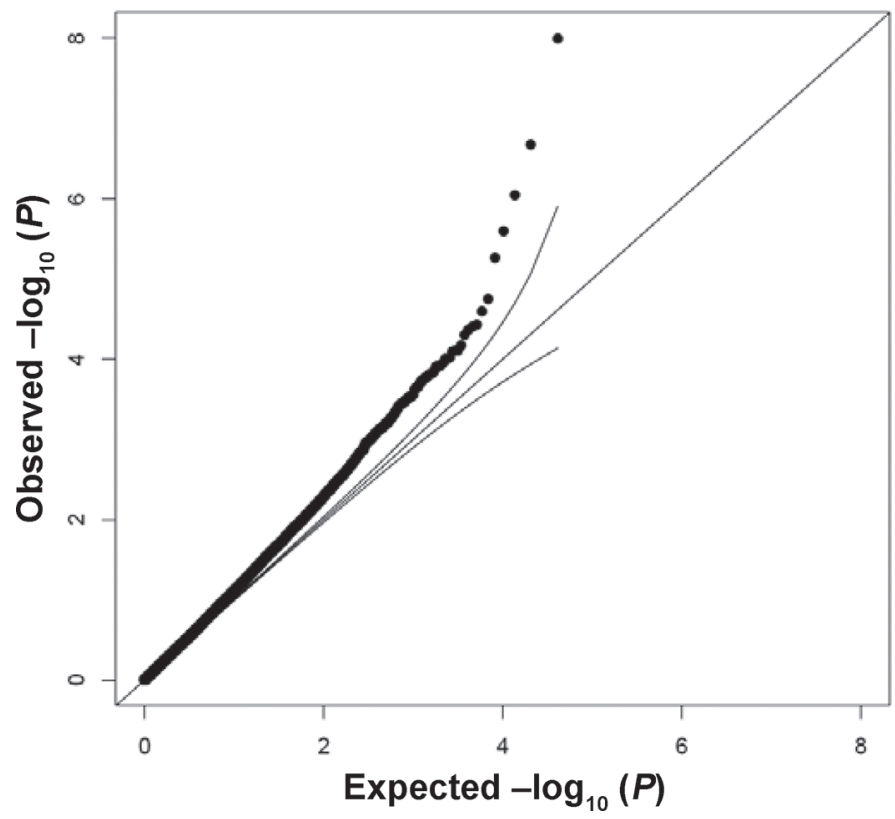

Figure 5. Quantile-quantile plot for binary distributed first testday fat-to-protein ratio after DGAT1 correction.

Overall, we identified 5 suggestively associated candidate SNP (pCD = 1e-04) influencing KET. For FPRbin (without DGAT1 correction), we detected 24 significant SNP $(\mathrm{pBF}=1.69 \mathrm{e}-06)$ and 30 suggestive SNP beyond the candidate threshold. For FPRbin (with DGAT1 correction), we detected 3 significantly associated (pBF $=1.69 \mathrm{e}-06)$ and 15 suggestive $\mathrm{SNP}(\mathrm{pCD}=1 \mathrm{e}-04)$. Despite the significant phenotypic associations and the moderate quantitative-genetic correlations between FPRbin and KET, different SNP were significantly associated with KET and FPR genomically.

\section{CONCLUSIONS}

Phenotypically, we detected strong associations between first test-day FPR and KET, as increasing KET incidences were significantly associated with higher FPR, and vice versa. In addition, we estimated moderate genetic correlations between KET and FPR using pedigree- or genomic-based relationship matrices. Results lead us to suggest the utilization of FPR from the first test-day as an indicator for genetic KET predictions. Heritabilities for KET, FPRgauss, and FPRbin were very similar. Interestingly, for KET, the estimate was slightly higher when modeling was based on the pedigree-based relationship matrix, but for FPRbin, higher estimates were achieved using the GRM. For FPRgauss, heritabilities were almost identical for both pedigree and genomic relationship matrices. Genomically, different SNP significantly contributed to KET and FPR, indicating different genetic mechanisms for both traits. We identified potential candidate genes being associated with diabetes and lipid metabolism, and which are involved in KET progression. Also, significantly associated SNP for FPR were in close distance to lipid and energy metabolism genes, influencing the state of NEB in early lactation.

\section{ACKNOWLEDGMENTS}

The authors gratefully acknowledge funding from the German Federal Ministry of Education and Research (BMBF, Bonn, Germany) and from the Forderverein Biookonomieforschung e.V. (FBV, Bonn, Germany)/ German Holstein Association (DHV, Bonn, Germany) for the collaborative project "KMU-innovativ-10: KuhL-Cow calibration groups for the implementation of selection strategies based on high-density genotyping in dairy cattle."

\section{REFERENCES}

Ali, A. K. A., and G. E. Shook. 1980. An optimum transformation for somatic cell concentration in milk. J. Dairy Sci. 63:487-490. https: //doi.org/10.3168/jds.S0022-0302(80)82959-6.

Bashir, S., I. Ashraf, S. Burkhari, and A. A. Dar. 2016. Clinical ketosis in lactating dairy cows and its therapeutic management. J. Livest. Sci. $7: 72-74$

Belay, T. K., M. Svendsen, Z. M. Kowalski, and T. Ådnøy. 2017. Genetic parameters of blood $\beta$-hydroxybutyrate predicted from milk infrared spectra and clinical ketosis, and their associations with milk production traits in Norwegian Red cows. J. Dairy Sci. 100:6298-6311. https://doi.org/10.3168/jds.2016-12458.

Bell, A. W. 1995. Regulation of organic nutrient metabolism during transition from late pregnancy to early lactation. J. Anim. Sci. 73:2804-2819. https://doi.org/10.2527/1995.7392804x.

Blanco-Gómez, A., S. Castillo-Lluva, M. Del Mar Sáez-Freire, L. Hontecillas-Prieto, J. H. Mao, A. Castellanos-Martín, and J. PérezLosada. 2016. Missing heritability of complex diseases: Enlightenment by genetic variants from intermediate phenotypes. BioEssays 38:664-673. https://doi.org/10.1002/bies.201600084.

Bovenhuis, H., M. H. P. W. Visker, N. A. Poulsen, J. Sehested, H. J. F. van Valenberg, J. A. M. van Arendonk, L. B. Larsen, and A. J. Buitenhuis. 2016. Effects of the diacylglycerol o-acyltransferase 1 (DGAT1) K232A polymorphism on fatty acid, protein, and mineral composition of dairy cattle milk. J. Dairy Sci. 99:3113-3123. https://doi.org/10.3168/jds.2015-10462.

Buttchereit, N., E. Stamer, W. Junge, and G. Thaller. 2012. Genetic parameters for energy balance, fat / protein ratio, body condition score and disease traits in German Holstein cows. J. Anim. Breed. Genet. 129:280-288. https://doi.org/10.1111/j.1439-0388.2011 $.00976 . x$.

Chen, S., H. Saiyin, X. Zeng, J. Xi, X. Liu, X. Li, and L. Yu. 2006. Isolation and functional analysis of human HMBOX1, a homeobox containing protein with transcriptional repressor activity. Cytogenet. Genome Res. 114:131-136. https://doi.org/10.1159/ 000093328.

Dai, J., C. Zhang, Z. Tian, and J. Zhang. 2011. Expression profile of HMBOX1, a novel transcription factor, in human cancers using highly specific monoclonal antibodies. Exp. Ther. Med. 2:487-490. https://doi.org/10.3892/etm.2011.240.

Duffield, T. F., K. D. Lissemore, B. W. McBride, and K. E. Leslie. 2009. Impact of hyperketonemia in early lactation dairy cows on 
health and production. J. Dairy Sci. 92:571-580. https://doi.org/ 10.3168/jds.2008-1507.

Dunnett, C. W. 1955. A multiple comparison procedure for comparing several treatments with a control. J. Am. Stat. Assoc. 50:10961121. https://doi.org/10.2307/2281208.

Eckel-Passow, J. E., D. J. Serie, B. M. Bot, R. W. Joseph, S. N. Hart, J. C. Cheville, and A. S. Parker. 2014. Somatic expression of ENRAGE is associated with obesity status among patients with clear cell renal cell carcinoma. Carcinogenesis 35:822-827. https://doi .org $/ 10.1093 /$ carcin/bgt485.

Ge, T., C.-Y. Chen, B. M. Neale, M. R. Sabuncu, and J. W. Smoller. 2017. Phenome-wide heritability analysis of the UK Biobank. PLoS Genet. 13:e1006711. https://doi.org/10.1371/journal.pgen .1006711

Golan, D., E. S. Lander, and S. Rosset. 2014. Measuring missing heritability: Inferring the contribution of common variants. Proc. Natl. Acad. Sci. USA 111:E5272-E5281. https://doi.org/10.1073/pnas .1419064111

Golay, A., and J. Ybarra. 2005. Link between obesity and type 2 diabetes. Best Pract. Res. Clin. Endocrinol. Metab. 19:649-663. https://doi.org/10.1016/j.beem.2005.07.010.

Gürtler, H., and F. J. Schweigert. 2005. Physiologie der Laktation. Physiologie der Haustiere, Enke Verlag, Stuttgart, Germany 2:552-573.

Hein, L., L. P. Sørensen, M. Kargo, and A. J. Buitenhuis. 2018. Genetic analysis of predicted fatty acid profiles of milk from Danish Holstein and Danish Jersey cattle populations. J. Dairy Sci. 101:2148-2157. https://doi.org/10.3168/jds.2017-13225.

Heringstad, B., Y. M. Chang, D. Gianola, and G. Klemetsdal. 2005. Genetic analysis of clinical mastitis, milk fever, ketosis, and retained placenta in three lactations of Norwegian Red cows. J. Dairy Sci. 88:3273-3281. https://doi.org/10.3168/jds.S0022 -0302(05)73010-1.

Heuer, C., Y. H. Schukken, and P. Dobbelaar. 1999. Postpartum body condition score and results from the first test day milk as predictors of disease, fertility, yield, and culling in commercial dairy herds. J. Dairy Sci. 82:295-304. https://doi.org/10.3168/jds.S0022 -0302(99)75236-7.

Kessel, S., M. Stroehl, H. H. D. Meyer, S. Hiss, H. Sauerwein, F. J. Schwarz, and R. M. Bruckmaier. 2008. Individual variability in physiological adaptation to metabolic stress during early lactation in dairy cows kept under equal conditions. J. Anim. Sci. 86:29032912. https://doi.org/10.2527/jas.2008-1016.

Kitamoto, T., A. Kitamoto, M. Yoneda, H. Hyogo, H. Ochi, T. Nakamura, H. Teranishi, S. Mizusawa, T. Ueno, K. Chayama, A Nakajima, K. Nakao, A. Sekine, and K. Hotta. 2013. Genome-wide scan revealed that polymorphisms in the PNPLA3, SAMM50, and PARVB genes are associated with development and progression of nonalcoholic fatty liver disease in Japan. Hum. Genet. 132:783792. https://doi.org/10.1007/s00439-013-1294-3.

Koeck, A., J. Jamrozik, F. S. Schenkel, R. K. Moore, D. M. Lefebvre, D. F. Kelton, and F. Miglior. 2014. Genetic analysis of milk betahydroxybutyrate and its association with fat-to-protein ratio, body condition score, clinical ketosis, and displaced abomasum in early first lactation of Canadian Holsteins. J. Dairy Sci. 97:7286-7292. https://doi.org/10.3168/jds.2014-8405.

Koeck, A., F. Miglior, J. Jamrozik, D. F. Kelton, and F. S. Schenkel. 2013. Genetic associations of ketosis and displaced abomasum with milk production traits in early first lactation of Canadian Holsteins. J. Dairy Sci. 96:4688-4696. https://doi.org/10.3168/jds $.2012-6408$

König, S., and K. May. 2018. Invited review: Phenotyping strategies and quantitative-genetic background of resistance, tolerance and resilience associated traits in dairy cattle. Animal 1-12. https:// doi.org/10.1017/S1751731118003208

König, S., A. R. Sharifi, H. Wentrot, D. Landmann, M. Eise, and H. Simianer. 2005. Genetic parameters of claw and foot disorders estimated with logistic models. J. Dairy Sci. 88:3316-3325. https:// doi.org/10.3168/jds.S0022-0302(05)73015-0.

Kroezen, V., F. S. Schenkel, F. Miglior, C. F. Baes, and E. J. Squires. 2018. Candidate gene association analyses for ketosis resistance in
Holsteins. J. Dairy Sci. 101:5240-5249. https://doi.org/10.3168/ jds.2017-13374.

Kurz, J. P., Z. Yang, R. B. Weiss, D. J. Wilson, K. A. Rood, G. E. Liu, and Z. Wang. 2019. A genome-wide association study for mastitis resistance in phenotypically well-characterized Holstein dairy cattle using a selective genotyping approach. Immunogenetics 71:35-47. https://doi.org/10.1007/s00251-018-1088-9.

Lau, W., T. Andrew, and N. Maniatis. 2017. High-resolution genetic maps identify multiple type 2 diabetes loci at regulatory hotspots in African Americans and Europeans. Am. J. Hum. Genet. 100:803-816. https://doi.org/10.1016/j.ajhg.2017.04.007.

Li, C., D. Sun, S. Zhang, S. Wang, X. Wu, Q. Zhang, L. Liu, Y. Li, and L. Qiao. 2014. Genome wide association study identifies 20 novel promising genes associated with milk fatty acid traits in Chinese Holstein. PLoS One 9:e96186. https://doi.org/10.1371/ journal.pone.0096186.

Li, M.-X., J. M. Y. Yeung, S. S. Cherny, and P. C. Sham. 2012. Evaluating the effective numbers of independent tests and significant p-value thresholds in commercial genotyping arrays and public imputation reference datasets. Hum. Genet. 131:747-756. https://doi .org/10.1007/s00439-011-1118-2.

Littlejohn, M. D., K. Tiplady, T. Lopdell, T. A. Law, A. Scott, C. Harland, R. Sherlock, K. Henty, V. Obolonkin, K. Lehnert, A. Macgibbon, R. J. Spelman, S. R. Davis, and R. G. Snell. 2014 Expression variants of the lipogenic AGPAT6 gene affect diverse milk composition phenotypes in Bos taurus. PLoS One 9:e85757. https://doi.org/10.1371/journal.pone.0085757.

Madsen, P., and J. Jensen. 2013. DMU: A Package for Analysing Multivariate Mixed Models. Accessed Mar 22, 2017. http://dmu.agrsci .dk/DMU/Doc/Current/dmuv6_guide.5.2.pdf.

McNeel, A. K., B. C. Reiter, D. Weigel, J. Osterstock, and F. A. Di Croce. 2017. Validation of genomic predictions for wellness traits in US Holstein cows. J. Dairy Sci. 100:9115-9124. https://doi.org/ $10.3168 /$ jds.2016-12323.

Momen, M., A. A. Mehrgardi, A. Sheikhy, A. Esmailizadeh, M. A. Fozi, A. Kranis, B. D. Valente, G. J. M. Rosa, and D. Gianola. 2017. A predictive assessment of genetic correlations between traits in chickens using markers. Genet. Sel. Evol. 49:16. https:// doi.org/10.1186/s12711-017-0290-9.

NCBI. 2017. PARVB parvin beta [Homo sapiens (human)]. Accessed Nov 5, 2017. https://www.ncbi.nlm.nih.gov/gene/29780.

Oguro, R., K. Kamide, T. Katsuya, H. Akasaka, K. Sugimoto, A. Congrains, Y. Arai, N. Hirose, S. Saitoh, M. Ohishi, T. Miura, and H. Rakugi. 2012. A single nucleotide polymorphism of the adenosine deaminase, RNA-specific gene is associated with the serum triglyceride level, abdominal circumference, and serum adiponectin concentration. Exp. Gerontol. 47:183-187. https://doi.org/10.1016/j .exger.2011.12.004

Oikonomou, G., K. Angelopoulou, G. Arsenos, D. Zygoyiannis, and G. Banos. 2009. The effects of polymorphisms in the DGAT1, leptin and growth hormone receptor gene loci on body energy, blood metabolic and reproductive traits of Holstein cows. Anim. Genet. 40:10-17. https://doi.org/10.1111/j.1365-2052.2008.01789.x.

Owen, K., and A. T. Hattersley. 2001. Maturity-onset diabetes of the young: From clinical description to molecular genetic characterization. Best Pract. Res. Clin. Endocrinol. Metab. 15:309-323. https: //doi.org/10.1053/beem.2001.0148.

Parker Gaddis, K. L., J. H. Megonigal, J. S. Clay, and C. W. Wolfe 2018. Genome-wide association study for ketosis in US Jerseys using producer-recorded data. J. Dairy Sci. 101:413-424. https://doi .org/10.3168/jds.2017-13383.

Patsenker, E., and F. Stickel. 2011. Role of integrins in fibrosing liver diseases. Am. J. Physiol. Gastrointest. Liver Physiol. 301:G425G434. https://doi.org/10.1152/ajpgi.00050.2011.

Preeti, K.-M., and K. J. Sushil. 2016. Hyperketonemia and ketosis increase the risk of complications in type 1 diabetes. Free Radic Biol. Med. 95:268-277. https://doi.org/10.1016/j.freeradbiomed .2016.03.020.

Pryce, J. E., K. L. Parker Gaddis, A. Koeck, C. Bastin, M. Abdelsayed, N. Gengler, F. Miglior, B. Heringstad, C. Egger-Danner, K. F. Stock, A. J. Bradley, and J. B. Cole. 2016. Invited review: 
Opportunities for genetic improvement of metabolic diseases. J. Dairy Sci. 99:6855-6873. https://doi.org/10.3168/jds.2016-10854.

Purcell, S., B. Neale, K. Todd-Brown, L. Thomas, M. A. R. Ferreira, D. Bender, J. Maller, P. Sklar, P. I. W. de Bakker, M. Daly, and P. Sham. 2007. PLINK: A tool set for whole-genome association and population-based linkage analyses. Am. J. Hum. Genet. 81:559575. https://doi.org/10.1086/519795.

Rask-Andersen, M., G. Moschonis, G. P. Chrousos, C. Marcus, G. V. Dedoussis, R. Fredriksson, and H. B. Schiöth. 2013. The STK33linked SNP rs4929949 is associated with obesity and BMI in two independent cohorts of Swedish and Greek children. PLoS One 8:e71353. https://doi.org/10.1371/journal.pone.0071353.

Robinson, A. M., and D. H. Williamson. 1980. Physiological roles of ketone bodies as substrates and signals in mammalian tissues. Physiol. Rev. 60:143-187. https://doi.org/10.1152/physrev.1980 .60.1.143.

Rui, L. 2014. Energy metabolism in the liver. Compr. Physiol. 4:177197. https://doi.org/10.1002/cphy.c130024.

Santos, L. V., K. Brügemann, A. Ebinghaus, and S. König. 2018. Genetic parameters for longitudinal behavior and health indicator traits generated in automatic milking systems. Arch. Tierzucht 61:161-171. https://doi.org/10.5194/aab-61-161-2018.

Schmatz, R., C. M. Mazzanti, R. Spanevello, N. Stefanello, J. Gutierres, P. A. Maldonado, M. Corrêa, C. S. da Rosa, L. Becker, M. Bagatini, J. F. Gonçalves, J. D. S. Jaques, M. R. Schetinger, and V. M. Morsch. 2009. Ectonucleotidase and acetylcholinesterase activities in synaptosomes from the cerebral cortex of streptozotocininduced diabetic rats and treated with resveratrol. Brain Res. Bull. 80:371-376. https://doi.org/10.1016/j.brainresbull.2009.08.019.

Segelke, D., J. Chen, Z. Liu, F. Reinhardt, G. Thaller, and R. Reents. 2012. Reliability of genomic prediction for German Holsteins using imputed genotypes from low-density chips. J. Dairy Sci. 95:54035411. https://doi.org/10.3168/jds.2012-5466.

Stock, K. F., J. Cole, J. Pryce, N. Gengler, A. Bradley, L. Andrews, B. Heringstad, and C. Egger-Danner. 2013. Standardization of health data. ICAR guidelines including health key. ICAR Tech. Ser. 17:75-81.

Suthar, V. S., J. Canelas-Raposo, A. Deniz, and W. Heuwieser. 2013. Prevalence of subclinical ketosis and relationships with postpartum diseases in European dairy cows. J. Dairy Sci. 96:2925-2938. https://doi.org/10.3168/jds.2012-6035.

Tetens, J., C. Heuer, I. Heyer, M. S. Klein, W. Gronwald, W. Junge, P. J. Oefner, G. Thaller, and N. Krattenmacher. 2015. Polymor- phisms within the APOBR gene are highly associated with milk levels of prognostic ketosis biomarkers in dairy cows. Physiol. Genomics 47:129-137. https://doi.org/10.1152/physiolgenomics .00126 .2014 .

van Knegsel, A. T. M., S. G. A. van der Drift, M. Horneman, A. P. W. de Roos, B. Kemp, and E. A. M. Graat. 2010. Short communication: Ketone body concentration in milk determined by Fourier transform infrared spectroscopy: Value for the detection of hyperketonemia in dairy cows. J. Dairy Sci. 93:3065-3069. https://doi .org/10.3168/jds.2009-2847.

Visscher, A., and S. Mason. 2016. DHI: Persistency of Milk Production. Accessed Mar 14, 2018. http://www.agromedia.ca/ADM Articles/content/DHI_persist.pdf.

White, H. M. 2015. The role of TCA Cycle anaplerosis in ketosis and fatty liver in periparturient dairy cows. Animals (Basel) 5:793-802. https://doi.org/10.3390/ani5030384.

Wu, G., K. Wang, Y. Xue, G. Song, Y. Wang, X. Sun, L. Zhong, C. Zhou, B. Shen, J. Chen, Y. Yu, H. Tang, Z. Peng, P. Sun, and X. Wang. 2016. Association of rs5764455 and rs6006473 polymorphisms in PARVB with liver damage of nonalcoholic fatty liver disease in Han Chinese population. Gene 575:270-275. https://doi .org/10.1016/j.gene.2015.09.007.

Yang, J., S. H. Lee, M. E. Goddard, and P. M. Visscher. 2011. GCTA: A tool for genome-wide complex trait analysis. Am. J. Hum. Genet. 88:76-82. https://doi.org/10.1016/j.ajhg.2010.11.011.

Yang, J., J. Zeng, M. E. Goddard, N. R. Wray, and P. M. Visscher. 2017. Concepts, estimation and interpretation of SNP-based heritability. Nat. Genet. 49:1304-1310. https://doi.org/10.1038/ng .3941 .

Zerbino, D. R., P. Achuthan, W. Akanni, M. R. Amode, D. Barrell, J. Bhai, K. Billis, C. Cummins, A. Gall, C. G. Girón, L. Gil, L. Gordon, L. Haggerty, E. Haskell, T. Hourlier, O. G. Izuogu, S. H. Janacek, T. Juettemann, J. K. To, M. R. Laird, I. Lavidas, Z. Liu, J. E. Loveland, T. Maurel, W. McLaren, B. Moore, J. Mudge, D. N. Murphy, V. Newman, M. Nuhn, D. Ogeh, C. K. Ong, A. Parker, M. Patricio, H. S. Riat, H. Schuilenburg, D. Sheppard, H. Sparrow, K. Taylor, A. Thormann, A. Vullo, B. Walts, A. Zadissa, A. Frankish, S. E. Hunt, M. Kostadima, N. Langridge, F. J. Martin, M. Muffato, E. Perry, M. Ruffier, D. M. Staines, S. J. Trevanion, B. L. Aken, F. Cunningham, A. Yates, and P. Flicek. 2018. Ensembl 2018. Nucleic Acids Res. 46:D754-D761. https://doi.org/10 $.1093 /$ nar/gkx1098. 